Review

\title{
CIK Cell Therapy for Solid Tumor
}

\author{
Jingjing Zhang ${ }^{1}$, Yao Yang ${ }^{2}$, Hualin $\mathrm{Fu}^{1}$ \\ ${ }^{1}$ Institute of Nano Biomedicine and Engineering, Department of Instrument Science and Engineering, Key Lab. for Thin Film and \\ Microfabrication Technology of Ministry of Education, School of Electronic Information and Electronic Engineering, Shanghai Jiao \\ Tong University, Shanghai 200240, China \\ ${ }^{2}$ School of Laboratory Medicine and Life Science, Wenzhou Medical University, Wenzhou, Zhejiang 325035, China \\ Corresponding author: E-mail: hfu@sjtu.edu.cn
}

Received: July. 15, 20I4; Accepted: Aug. 15, 20I4; Published: Aug. 29, 2014.

Citation: Jingjing Zhang, Yao Yang and Hualin Fu. CIK Cell Therapy for Solid Tumor. Nano Biomed. Eng. 20I4, 6(2), 60-66. DOI: $10.5101 /$ nbe.v6i2.p60-66.

\begin{abstract}
In recent years, the clinical trials on applying cytokine induced killer (CIK) cells to solid tumor therapy have been launched worldwide. CIK cells are a heterogeneous subset of ex-vivo expanded $\mathrm{T}$ lymphocytes, they present a mixed T-NK phenotype and are endowed with a MHC-unrestricted antitumor activity, which have showed great advantages in clinical treatment compared with other adoptive immunity therapies, therefore, they are more and more widely used in adoptive immunotherapy of tumors. CIK cells can be used alone or in combination with surgery radiotherapy and chemotherapy for comprehensive treatment. In the present article, we keep a track of research progress both home and abroad, review the main functional characteristics of CIK cells, briefly introduce the source phenotype activation and amplification of the CIK cells, and summarize their targeting and anti-tumor mechanisms new techniques and methods and current status of international clinical trials from several aspects.
\end{abstract}

Keywords: Cytokine Induced Killer Cells; Cancer; Clinical Trial; Cell Immunotherapy

\section{Introduction}

There are several ways for the treatment of solid tumors recent years, and adoptive immunotherapy holds great promise in the scenario of potential new approaches refractory to conventional therapies. Crucial issues for all adoptive immunotherapy strategies include the obtainment of sufficient numbers of immune effectors, recognition of tumor targets and possible restriction to specific HLAhaplotypes. Cytokine-induced killer (CIK) cells were first reported in the world by Schmidt-Wolf [1]for its high proliferative capacity and high cytotoxicity in 1991. Compared with LAK and TIL cells, CIK cells demonstrate a stronger activity of tumor destruction.
This paper introduces and summarizes the basic and clinical research for the treatment of malignant tumor by CIK cells and raises some problems, which provide an evidence for CIK cell therapy for cancer in the future.

\section{The targeting and anti-tumor mechanism of CIK cells \\ Tumor targeting characteristic of CIK cells}

CIK cells are equipped with tumor targeting ability, homing to the tumor site when injected to the normal tissues or through vascular perfusion. 


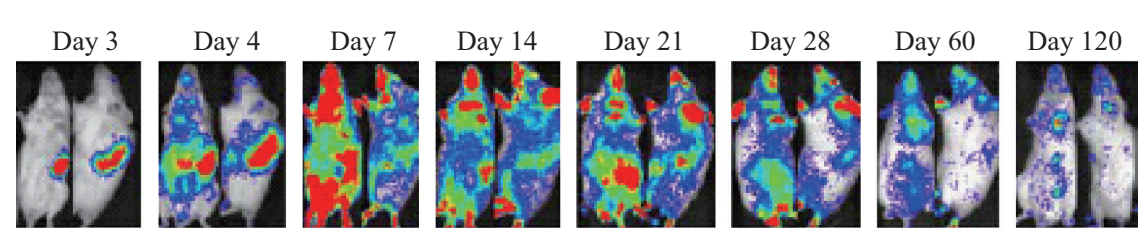

Fig. 1 CIK cells homing to tumor site [2].

Some scholars abroad have taken advantage of the characteristic of CIK cells to establish a "vehicle", carrying virus accurately locate to tumor tissue in the immunodeficiency mice model. In China, there are fewer studies about the trend migration to tumor of CIK cells, and we have mainly focused on the study of distribution pattern of CIK cells in the body.

The team of Ryosei Nishimura had evaluated CIK cell accumulation into tumor sites in their research in 2008 [2]. They found that the donor derived CIK cells in the allogeneic mice homed to and proliferated in secondary lymphoid organs, followed by the infiltration of gut and skin (Fig. 1). The similar work has also been done by a Chinese research team, who had established the xenografts in nude mice model, injected the fluorescent dye CIK cells labeled Dil to the nude mice through the tail vein, and observed the trend migration to tumor of CIK cell in vivo imaging system. The result also showed the phenomena of tumor targeting.

Though lots of data have shown that CIK cells did home to the tumor tissue, there are no clear evidences explaining the result. Some antigen molecules expressed on the surface of CIK cells are considered to be related to the recognition of tumor cells. For example, the NK cell receptors like NKG2D, and the $\mathrm{T}$ cell receptors like TCR, respectively bind to the molecules expressed on tumor cells.

\section{Anti-tumor mechanism of ClK cells}

The ability to efficiently kill tumor cells is the ultimate basic ability requested to immune effectors candidate for adoptive immunotherapy. Researchers home and abroad have confirmed the killing ability of CIK cells by lots of experiments (Fig. 2). CIK cells, a kind of heterogeneous cells, endowed with a MHCindependent tumor killing capacity against both solid and hematologic malignancies, are a mixed cell group which contains $\mathrm{CD}^{+} \mathrm{CD}^{+} 6^{+}$and $\mathrm{CD} 3^{+} \mathrm{CD} 8^{+}$cells and the main effect cells are $\mathrm{CD}^{+} \mathrm{CD}^{+} 6^{+}$cells. The antitumor activity is mainly, even if not exclusively, associated with the $\mathrm{CD}^{+} \mathrm{CD}^{+} 6^{+}$fraction [3]. $\mathrm{CD}^{+}$ CD56 $6^{+}$cells only contains $1 \% \sim 5 \%$ in peripheral blood lymphocytes, however, the cells can be acquired rapidly under the certain condition of the cultivation, generally the quantity of the cells can reach more than 1000 times after $20 \mathrm{~d}$ cultivation in vitro, and when we inject the cells back to the body, with the help of IL2 , they can still be further proliferated. There are still evidence shows that in the process of culturing CIK cells, $\mathrm{CD}^{+}$cells, that is T cells, amplify rapidly, while the anti-T cells are eliminated gradually. Pievani etc. purified the mononuclear cells after 2 weeks cultivation by using the immune magnetic beads technology [4], confirmed that the $\mathrm{CD}^{+} \mathrm{CD} 56^{+} \mathrm{CIK}$ cells account for $85 \%$ of the total number of all the culture cells. According to the method above, we could get a large quantity of CIK cells, which can be applied to clinical therapy in malignant tumor.

At present, it is considered that the anti-tumor mechanism of CIK cells may have the following aspects:

(1) When CIK cells are activated, the combination capacity of lymphocyte function associated antigen (LFA-1) and intercellular surface adhesion molecule-1 (ICAM-1) is transformed from low affinity to high affinity, discharging toxic particles such as n-methyl carbonyl sulfur - left-handed - lysine methyl ester.

(2) CIK cells may also be activated by the combination of $\mathrm{CD}^{+}$and its receptor on the cell surface, $\mathrm{CD}^{+}{ }^{+}$connected with $\mathrm{T}$ cell receptor (TCR)

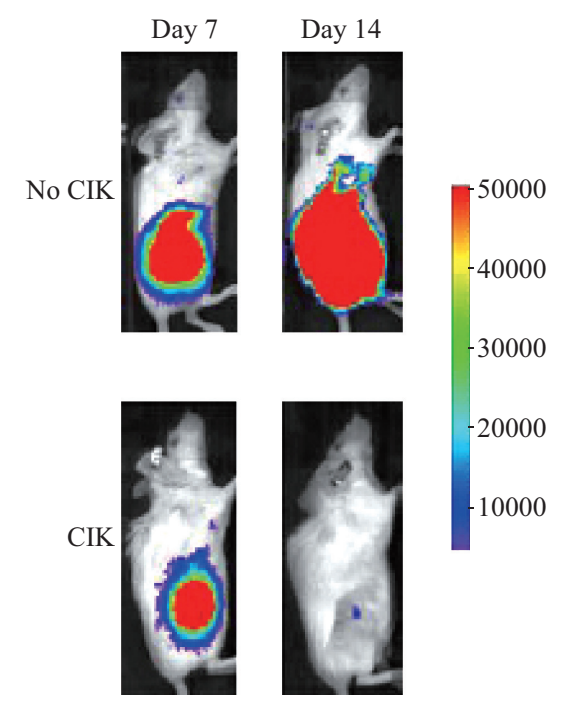

Fig. 2 The tumor killing ability of CIK cells [2]. 
participate in the signal transmission, also triggers the release of the toxicity of cytoplasmic granules to produce soluble tumor effect.

(3) CIK cells themselves can secrete cytokines like IL-2, IL-6, TNF and GM-CSF, which can kill target cells alone or raise the activity of effect cells in high concentrations relatively.

(4) CIK cells can also induce the apoptosis of tumor cells. CIK cells can activate apoptosis genes in tumor cell, improving the gene expression of FLIP, Bcl-2, Bcl-xl, DAD1 and surviving [5].

(5) CIK cells express much more FasL (Fas ligand), which can couple to the Fas expressed on the surface of tumor, induce the apoptosis of tumor cells.

(6) The NKG2D molecule expressed on the surface of CIK cells helps to recognize and kill the tumor cells, by interacting with MHC-unrestricted ligands on tumor cells [6]. NKG2D belong to the c-type lectin activating receptor family and it is located within the NK gene complex [7]. NKG2D has a co-stimulatory role on conventional $\mathrm{T}$ lymphocytes, while on CIK cells it acquires a tumor-receptor function, following the exvivo activation with IL-2 and up-regulation of the DAP10 adaptor molecule [6], its artificial inhibition with blocking Abs or siRNA experiments confirmed that CIK-induced cytolysis is mediated by NKG2D rather than T-cell receptor (TCR) Besides, NKG2D, NKp30, DNAM-1 and the LFA-1/ICAM-1 complex also have been recently described as implicated in tumor recognition [7]. The main ligands recognized by NKG2D are MHC related molecules A and B, stressinducible proteins expressed, almost exclusively, by malignant tumor cells regardless of the original histotype [4].

\section{The preparation of CIK cells}

\section{The cellular characteristics of CIK cells}

One of the important limitations in clinical trials of several adoptive immunotherapy strategies is the obtainment of enough numbers of anti-tumor immune effectors and their invivo persistence after infusion into the patients. It is worth mentioning that, the important and positive characteristics of CIK cells are their easy, and relatively inexpensive, exvivo expansion [8]. CIK cells can come from autogenous and allogeneic normal tissues or from different tissues in one body, which makes them the relatively convenient way to get.

\section{CIK cells from autogenous and allogeneic normal tissues}

Patients with malignant tumor have different degrees of impaired immune function; the number of $\mathrm{CD}^{+} \mathrm{CD}^{+} \mathrm{CD} 8^{+}$cells is obviously reduced. The ways treating patients with autologous CIK cells expanded in vitro show great security, can avoid other diseases caused by cross infection. Researchers like SchmidtWolf treated tumor patients by CIK cells induced from their own CIK cells in peripheral blood lymphocyte, finding that the interferon (IFN) in serum, granulocyte-macrophage colony-stimulating factor (GM-CSF) and TNF levels increased significantly in patients during treatment and the proportion of $\mathrm{CD}^{+}$cells in peripheral blood in patients increased as well. However, as we all know, cancer patients are equipped with immune tolerance in different degrees, and the more serious the patients are, the more obviously decline of their immune function. So the application of allogeneic CIK cells replacing the patient's own CIK cells become one of the ways to solve this problem. And sometimes in terms of activation and amplification, allogeneic CIK cells are far superior to CIK cells from patients themselves.

\section{CIK cells from different tissues}

CIK cells precursors are $\mathrm{CD}^{+} \mathrm{T}$ lymphocytes, they can be not only classically expanded starting from peripheral blood mono-clear cells (PBMC) but may also be generated from bone marrow or umbilical cord blood precursors [9]. In order to obtain the best quality of CIK cells, we can choose appropriate ways and methods to get the CIK cells according to the actual situation.

\section{CIK cells from peripheral blood lymphocytes (PBL)}

CIK cells can be generated by in vitro expansion of peripheral blood lymphocytes (PBL) with anti-CD antibodies, IL-2 and other cytokines. This is a relatively general way to obtain abundant number of CIK cells. In such culture, more than 1000 fold expansion of cells can be achieved over 21-day in vitro culture. 
Among expanded CIK cells, the cells with the greatest cytotoxicity against tumor cell lines express both the T-cell marker CD3 and the NK cell marker CD56. Though $\mathrm{CD}^{+} \mathrm{CD}^{+} 6^{+}$cells are rare in uncultured PBLs, consistent with the phenotype of resting naïve and memory $\mathrm{T}$ cells, after culture in this way, $\mathrm{CD}^{+} \mathrm{CD}^{+} 6^{+}$effector cells can be propagated in a shorter time.

\section{CIK cells from umbilical cord blood}

Umbilical cord blood have advantages of alternative sources, weak immunogenicity, easy ways to take and rich in hematopoietic stem cells and mononuclear cells, and the content of CD $34^{+}$cells in umbilical cord blood are higher and purer which equipped with higher capability of proliferation than the cells from bone marrow and peripheral blood. After culturing, the $\mathrm{CD}^{+}$ $\mathrm{CD}^{2} 6^{+}$cells in umbilical cord blood (the main effector of CIK cells) amplified more than 900 times, and $\mathrm{CD}^{+} \mathrm{CD}^{+} \mathrm{T}$ cells increased significantly, $\mathrm{CD} 3^{+} \mathrm{CD} 16^{+} \mathrm{CD}^{+} 6^{+}$cells increased in the same time, the results showed that umbilical cord blood can produce CIK cells and amplify potential is extremely strong, the killing activity of amplified CIK cells is also higher than peripheral blood CIK cells [10]-[13]. Comprehensive the above research results, that is derived from umbilical cord blood CIK cells is more suitable for clinical treatment.

\section{CIK cells from bone marrow}

The proliferation ability of CIK cells from bone marrow is a bit poorer than CIK cells from peripheral blood, but there are no significant differences in cell toxicity, compared with peripheral blood CIK cells. Linn, etc. had made a collection of bone marrow samples from patients with acute myelocytic leukemia to induce CIK cells, and found that CIK cells obtained in this way is not much difference in activation, amplification and the capability of killing target cells.

In conclusion, these four kinds of ways can be referred to as a source of precursor cells of CIK cells to collect and culture. Before treatment we should choose the appropriate acquisition methods according to the actual circumstances of the laboratory, training experience, especially the willingness of the patients and family members.

\section{The role of biomolecules in CIK cells culture}

\section{The effect of Dendritic Cell}

Many laboratories use Dendritic Cell (DC) and CIK cells co-culture in order to improve the treatment effect:

(1) DC is a kind of strong antigen presenting cells, it can present information of antigen to $\mathrm{T}$ cells, which significantly enhance the activity of CIK cells; (2) After $24 \mathrm{~h}$ mixed culture with DC and CIK cells, the secretion of IL-12 amount to 6.93 times of CIK cells in separate culture; (3) DC antigen presenting function can extend the time of secreting IFN- $\gamma$ of CIK cells and increase the production of IFN- $\gamma, 3 \sim 5$ times that of pure CIK cell culture; (4) Increase $\mathrm{CD} 3^{+}, \mathrm{CD}^{+}$and $\mathrm{CD}^{+} \mathrm{CD}^{+}$and $\mathrm{CD}^{+} \mathrm{CD}^{+} 6^{+}$cells and decrease $\mathrm{CD} 4^{+}$ $\mathrm{CD} 25^{+}$Treg cells; (5) The associated antigen of tumor is obviously drop, and the life quality of patients with tumor improved significantly; (6) The efficiency of killing tumor is higher than that of pure CIK cells in the treatment group, and no obvious toxic effect.

\section{The role of cytokines}

IL-2, IL-7, IL-15 play, an important role in maintaining the cytotoxic activity of killer cells. Growth factor from stem cell can be coordinated with IL-2 promoting the cell proliferation to increase the stimulation effect of IL-2. Compared with the effect of applying IL-15, the synergy of FMS tyrosine kinase-3 ligand and IL-15 can significantly increase the number of $\mathrm{CD} 34^{+} \mathrm{NK}$ cells, and meanwhile it can increase the CD34 ${ }^{+}$hematopoietic stem cells on the expression of IL-2/IL-15 R. The latest research report also showed that IL-15 can effectively augment the number of culture CIK cells, in the treatment of acute myeloid leukemia, leiomyosarcoma and rhabdomyosarcoma achieved encouraging results.

\section{The role of the thymus globulin}

Thymus globulin (TG) is a kind of polyclonal antibody IgG. It is generally believed the thymus globulin can replace CD3 monoclonal antibody and combine with IFN IL-2, achieving the goal of effectively amplify CIK cells. The researchers assessed the ability of killing target cells of CIK cells induced by thymus globulin, results showed that the cells can be more effective in killing K562 tumor cells, and can 
Table 1 Published studies of CIK clinical trials in adoptive immunotherapy

\begin{tabular}{|c|c|c|c|c|c|}
\hline Disease & Patients & Type of CIKs & Toxicity & Clinical responses & Ref. \\
\hline $\begin{array}{l}\text { Colorectal and } \\
\text { renal carcinoma }\end{array}$ & 10 & Autologous & Fever: 3 & CR (1) SD (3) & [14] \\
\hline $\begin{array}{c}\text { NHL; Renal } \\
\text { carcinoma; HCC }\end{array}$ & 12 & Autologous & Fever: 2 & $\mathrm{CR}(3) ; \mathrm{SD}(2)$ & {$[15]$} \\
\hline Advanced NSCLC & 59 & $\begin{array}{c}\text { Autologous } \\
(+ \text { Chemotherapy) }\end{array}$ & None relevant & Improved PFS and OS & [16] \\
\hline HCC (adjuvant setting) & 85 & Autologous & None relevant & Reduced recurrence rate & [17] \\
\hline $\begin{array}{l}\text { Gastric cancer } \\
\text { (Stage IV) }\end{array}$ & 57 & $\begin{array}{c}\text { Autologous } \\
(+ \text { Chemotherapy) }\end{array}$ & None relevant & $\begin{array}{c}\text { Reduced Tumor Markers; Improved } \\
\text { QOL }\end{array}$ & {$[18]$} \\
\hline $\mathrm{HCC}$ & 13 & Autologous & $\begin{array}{l}\text { Transient Fever } \\
\text { (most of pts) }\end{array}$ & $\begin{array}{l}\text { Reduced tumor volume (3); } \\
\text { Im-proved symptoms; } \\
\text { Decreased HBV-DNA load }\end{array}$ & [19] \\
\hline
\end{tabular}

produce a lot of active IL-12 p40.

\section{Clinical Studies}

The clinical translation of adoptive immunotherapy with CIK cells as treatment for patients with malignant tumors is currently the key of question of the clinical trials and their number has increased in the very recent years. Here are two trials with CIK cells for solid tumors that have currently been published in Europe or U.S. One is the clinical experience [14] included 10 patients with metastatic renal carcinoma, colorectal cancer and lymphoma. Circulating CIK cells persisted for up to 2 weeks after the infusion, and there is no relevant associated toxicities were found. The results turn out that one patient with lymphoma got a complete response, compared to the six patients had progressive diseases and three did not experience any change. The other important experience is to confirm the safety and feasibility of this immunotherapy approach along with demonstration of initial clinical activity. The clinical translation of adoptive immunotherapy with CIK cells as treatment for patients with solid tumors is currently the object of clinical trials and their number has increased in the very recent years. Nowadays a higher number of clinical studies have been recently performed. Table 1 represents some clinical trials with CIK cells for the treatment of solid tumors.

The studies above confirmed the high safety of CIK cells applied in adoptive immunotherapy which suggested its clinical activities. It is difficult to draw a clear statement about tumor response or impact on survival for the heterogeneity in methods and standards of response assessment. Recently, it has created an international registry of CIK cells (IRCC) to collect data and report the results about the CIK therapy for cancer on a global scale, which makes great help to assess the response of the experiment and provide a useful tool to analyze past and future results, is crucial to obtain objective and reliable conclusion on the effect of CIK cells as adoptive immunotherapy for cancer.

\section{The existing problems and prospect for the future}

Cancer immunotherapy has been considered a promising approach for a very long time and CIK cells represent a realistic tool in the scenario of cancer adoptive immunotherapy strategies. Their easy and inexpensive ex-vivo expansion, along with the MHC-unrestricted tumor killing ability may overcome some crucial problems which have limited the diffusion and clinical translation of other immunotherapy approaches. That is to say CIK cells offer a safe and effective approach to treat the malignant tumor.

CIK cells have been applied in some areas like leukemia, lymphoma and solid tumor treatment; however, the treatment effect is limited. An effective way to solve the problem is the genetic engineering modification of CIK cells. To achieve the purpose of removal of tumor is need to get a lot of CIK cells. Foreign scholars such as Marin have delivered encoded anti-CD33-C and anti-CD33-CCD28-OX4-C receptor gene SFC retrovirus to CIK cells, results show that the transgenic cells have greatly improved the anti-leukemia activity, 
and increased cell killing activity to different sources of acute myelogenous leukemia, and can release high levels of cytokines.

In terms of the clinic trials, there still exist some problems. In recent years, clinical trials about the application of CIK cells for solid tumor therapy are carried out at home and abroad. Internationally, Schmidt-Wolf at Stanford university in California first reported the CIK cells treatment, other European countries, such as Britain, Italy, France, Germany, Asia such as Japan, South Korea, Singapore, India and Australia and the other countries have successively reported basic research and clinical trials. However, compared to the international countries, China is the country who have carried out the most of clinic trials, and published the most of papers. CIK cells treatment first applied in domestic cities such as Beijing, Shanghai, Tianjin, Harbin, Guangzhou, Shenzhen, which have a large number of reports of clinical application. Why such a big difference of CIK therapy practice between Chinese and western countries? I think the reasons can be summarized as follows: (1) First of all, thanks to the national policy, CIK cell therapy was referred to as a third category of clinical technology by the ministry of health; (2) The second, China's population base is so big that lead to a high rates of tumor; (3) In addition to the public hospital, part of the army universities and hospital are open to the society; (4) Medical market makes the international capital and some domestic companies, investors and so on are racing to develop the areas; (5) Some organization driven by interests, without any basic research, even in the absence of the basic theory and practice, direct transition to the clinical treatment; (6) Medical management regulation is missing or not in place with other technical problems, for example, a lot of clinical research have no effective register, are lack of academic argumentation and high quality clinical trial scheme, resulting in unconvincing clinical data and having difficulty in solving some specific clinical practical problems. According to the problem above, the researchers in the future should take all these into consideration and make a great effort to develop the CIK cell therapy for cancer.

\section{References}

[1] Schmidt-Wolf IG, Negrin RS, Kiem HP, Blume KG, Weissman IL: Use of a SCID mouse/human lymphoma model to evaluate cytokine-induced killer cells with potent antitumor cell activity. The Journal of experimental medicine 1991, 174(1): 139-149.

[2] Nishimura R, Baker J, Beilhack A, Zeiser R, Olson JA, Sega EI, Karimi M, Negrin RS: In vivo trafficking and survival of cytokine-induced killer cells resulting in minimal GVHD with retention of antitumor activity. Blood 2008, 112(6): 2563-2574.

[3] Sangiolo D, Martinuzzi E, Todorovic M, Vitaggio K, Vallario A, Jordaney N, Carnevale-Schianca F, Capaldi A, Geuna M, Casorzo L et al.: Alloreactivity and anti-tumor activity segregate within two distinct subsets of cytokineinduced killer (CIK) cells: implications for their infusion across major HLA barriers. International immunology 2008, 20(7): 841-848.

[4] Pievani A, Borleri G, Pende D, Moretta L, Rambaldi A, Golay J, Introna M: Dual-functional capability of CD3+CD56+ CIK cells, a T-cell subset that acquires NK function and retains TCR-mediated specific cytotoxicity. Blood 2011, 118(12): 3301-3310.

[5] Verneris MR, Kornacker M, Mailander V, Negrin RS: Resistance of ex vivo expanded $\mathrm{CD}^{+}{ }^{+} \mathrm{CD} 56^{+} \mathrm{T}$ cells to Fas-mediated apoptosis. Cancer immunology, immunotherapy : CII 2000, 49(6): 335-345.

[6] Verneris MR, Karami M, Baker J, Jayaswal A, Negrin RS: Role of NKG2D signaling in the cytotoxicity of activated and expanded CD8 ${ }^{+}$T cells. Blood 2004, 103(8): 30653072 .

[7] Houchins JP, Yabe T, McSherry C, Bach FH: DNA sequence analysis of NKG2, a family of related cDNA clones encoding type II integral membrane proteins on human natural killer cells. The Journal of experimental medicine 1991, 173(4): 1017-1020.

[8] Cosman D, Müllberg J, Sutherland CL, Chin W, Armitage R, Fanslow W, Kubin M, Chalupny NJ: ULBPs, Novel MHC Class I-Related Molecules, Bind to CMV Glycoprotein UL16 and Stimulate NK Cytotoxicity through the NKG2D Receptor. Immunity 2001, 14(2): 123-133.

[9] Lu PH, Negrin RS: A novel population of expanded human $\mathrm{CD} 3{ }^{+} \mathrm{CD} 56^{+}$cells derived from $\mathrm{T}$ cells with potent in vivo antitumor activity in mice with severe combined immunodeficiency. Journal of immunology (Baltimore, Md: 1950) 1994, 153(4): 1687-1696.

[10] Cohen SB, Woolley J, Bogunia-Kubik K, Natarajan P, Kotecha R, Belaramani L, Fallen PR, Perez-Cruz I, Madrigal JA: Macrophage colony stimulating factor (M-CSF) within cord blood sera may be partially responsible for the reduced proliferation of cord blood $\mathrm{T}$ cells. European cytokine network 2000, 11(4): 608-617.

[11] Robinson KL, Ayello J, Hughes R, van de Ven C, Issitt L, Kurtzberg J, Cairo MS: Ex vivo expansion, maturation, and activation of umbilical cord bloodderived T lymphocytes with IL-2, IL-12, anti-CD3, and IL-7. Potential for adoptive cellular immunotherapy post-umbilical cord blood transplantation. Experimental hematology 2002, 30(3): 245-251.

[12] Gluckman E, Rocha V, Chevret S: Results of unrelated umbilical cord blood hematopoietic stem cell transplant. Transfusion clinique et biologique: Journal de la Societe francaise de transfusion sanguine 2001, 8(3): 146-154.

[13] Ende N, Lu S, Alcid MG, Chen R, Mack R: Pooled umbilical cord blood as a possible universal donor for marrow reconstitution and use in nuclear accidents. Life sciences 2001, 69(13): 1531-1539. 
[14] Schmidt-Wolf IG, Finke S, Trojaneck B, Denkena A Lefterova P, Schwella N, Heuft HG, Prange G, Korte $\mathrm{M}$, Takeya $\mathrm{M}$ et al.: Phase I clinical study applying autologous immunological effector cells transfected with the interleukin-2 gene in patients with metastatic renal cancer, colorectal cancer and lymphoma. British journal of cancer 1999, 81(6): 1009-1016.

[15] Olioso P, Giancola R, Di Riti M, Contento A, Accorsi P, Iacone A: Immunotherapy with cytokine induced killer cells in solid and hematopoietic tumours: a pilot clinical trial. Hematological oncology 2009, 27(3): 130-139.

[16] Wu C, Jiang J, Shi L, Xu N: Prospective study of chemotherapy in combination with cytokine-induced killer cells in patients suffering from advanced non-small cell lung cancer. Anticancer research 2008, 28(6b): 39974002.

[17] Weng DS, Zhou J, Zhou QM, Zhao M, Wang QJ, Huang LX, Li YQ, Chen SP, Wu PH, Xia JC: Minimally invasive treatment combined with cytokine-induced killer cells therapy lower the short-term recurrence rates of hepatocellular carcinomas. Journal of immunotherapy (Hagerstown, Md : 1997) 2008, 31(1): 63-71.

[18] Jiang J, Xu N, Wu C, Deng H, Lu M, Li M, Xu B, Wu J, Wang R, Xu J et al.: Treatment of advanced gastric cancer by chemotherapy combined with autologous cytokineinduced killer cells. Anticancer research 2006, 26(3b): 2237-2242.

[19] Shi M, Zhang B, Tang ZR, Lei ZY, Wang HF, Feng YY, Fan ZP, Xu DP, Wang FS: Autologous cytokine-induced killer cell therapy in clinical trial phase I is safe in patients with primary hepatocellular carcinoma. World journal of gastroenterology: WJG 2004, 10(8): 1146-1151.

Copyright $(2014$ Jingjing Zhang, Yao Yang and Hualin Fu. This is an open-access article distributed under the terms of the Creative Commons Attribution License, which permits unrestricted use, distribution, and reproduction in any medium, provided the original author and source are credited. 